\title{
Radical cystectomy: a review of techniques, developments and controversies
}

\author{
Khatereh Aminoltejari, Peter C. Black \\ Department of Urologic Sciences, Faculty of Medicine, The University of British Columbia, Vancouver, BC, Canada \\ Contributions: (I) Conception and design: K Aminoltejari; (II) Administrative support: PC Black; (III) Provision of study material or patients: None; \\ (IV) Collection and assembly of data: K Aminoltejari; (V) Data analysis and interpretation: None; (VI) Manuscript writing: All authors; (VII) Final \\ approval of manuscript: All authors. \\ Correspondence to: Dr. Peter C. Black. Gordon and Leslie Diamond Health Care Centre, 6th Floor, 2775 Laurel Street, Vancouver General Hospital, \\ Vancouver, BC V5Z 1M9, Canada. Email: pblack@mail.ubc.ca.
}

\begin{abstract}
Radical cystectomy (RC) with urinary diversion is considered the standard treatment for muscle invasive bladder cancer (MIBC). As one of the most challenging surgical techniques performed by urologists, $\mathrm{RC}$ was described many decades ago, and yet patient morbidity rates have remained stagnant over the years. This review outlines the most recent indications and techniques for RC and analyses the current landscape of complications after cystectomy. There is significant room for improvement with respect to both oncologic and functional outcomes after RC. Future efforts will need to focus on unifying reporting methodology, optimal patient selection criteria, enhanced surgical techniques and peri-operative care pathways, and technological advances to improve patient outcomes.
\end{abstract}

Keywords: Complications; pelvic lymph node dissection (PLND); radical cystectomy (RC); urothelial carcinoma of bladder

Submitted Nov 13, 2019. Accepted for publication Mar 04, 2020.

doi: $10.21037 /$ tau.2020.03.23

View this article at: http://dx.doi.org/10.21037/tau.2020.03.23

\section{Introduction}

Urothelial carcinoma of the bladder (UCB) (1) is the fourth most common cancer in men, and the fifth most common cancer overall according to 2019 statistics reported by the American Cancer Society (1). Approximately 80,470 new cases of UCB (61,700 in men and 18,770 in women) and 17,670 deaths from UCB (12,870 in men and 4,800 in women) are expected in 2019 (1). UCB accounts for approximately $90 \%$ of all bladder malignancies. Although most patients present with non-muscle invasive bladder cancer (NMIBC), approximately $30 \%$ of patients present with muscle invasive bladder cancer (MIBC) and that number goes up to $40 \%$ when considering patients who progress from NMIBC to MIBC (2-4).

Radical cystectomy (RC) plays a central role in the treatment of UCB (3). It is a mainstay in the management of MIBC, together with peri-operative chemotherapy and appropriate lymphadenectomy, but is also important for the treatment of very high risk and treatment-refractory NMIBC (5-7). RC is one of the most challenging surgical techniques in Urology. Although the first records of RC date back to the 1900s, Marshall and Whitmore were the first to describe the surgical principles of RC in the 1940s. These same techniques have been refined over time, but complication rates and morbidity remain high after RC (8). Minimally invasive techniques including robotic-assisted laparoscopic RC have been developed, but these have not yet significantly impacted outcomes (3).

\section{Indications for RC}

RC can be performed for NMIBC, MIBC or metastatic UCB (mUCB) (9). In the NMIBC setting, RC is most commonly performed for high risk disease (high grade Ta/ T1 tumors of carcinoma in situ (CIS) that has recurred or 
persisted after adequate intravesical Bacillus CalmetteGuerin (BCG) therapy. The risk of progression to MIBC is high in this situation, salvage treatment options are otherwise relatively ineffective, and RC represents a highlight curative intervention. High grade recurrences after induction BCG (if recurrence is T1) or induction and one round of maintenance BCG (if recurrence is Ta or CIS) are considered "BCG-unresponsive", in addition to high grade tumors that recur within 6 months $(\mathrm{Ta} / \mathrm{T} 1)$ or 12 months (CIS) after the last dose of BCG if there has been a previous disease-free period (10). RC is the standard of care for BCG-unresponsive high risk NMIBC $(11,12)$.

$\mathrm{RC}$ is also indicated as first line therapy (i.e., without prior BCG therapy) of very high risk NMIBC. Features that make a NMIBC very high risk include: presence of lymphovascular invasion, variant histology (e.g., micropapillary, sarcomatoid, or plasmacytoid histology), extensive invasion into the lamina propria, large high grade T1 tumors $(>5 \mathrm{~cm})$, persistent T1 tumor on re-resection and presence of disease in the prostatic urethra $(11,12)$.

Optimal treatment of non-metastatic MIBC (T2T4N0M0) consists of neoadjuvant cisplatin-based chemotherapy followed by RC (2). In select patients trimodal therapy (TMT), which includes maximal transurethral resection of bladder tumour (TURBT) and chemoradiation $(13,14)$, is an alternative to RC. RC is also a standard component of TMT protocols should there be persistence or recurrence of tumor in the bladder (Figure 1). Importantly, approximately half of patients in the U.S. $(3,15)$ with non-metastatic MIBC do not receive any treatment with curative intent. In a systematic review by Williams and colleagues [2019], RC was used as a mode of therapy in $6 \%$ of patients across all age groups and $19-21 \%$ among patients who were 66 years old or younger (5). An important initiative moving forward will be increase the number of patients receiving treatment with curative intent, whether it be TMT or RC (15).

RC plays a limited role in locally advanced or mUCB. Patients with unresectable local disease, which is defined as clinical stage T4b or N1-3, are usually treated primarily with chemotherapy, which is followed by RC in patients with a favorable response. Dodd and colleagues in 1999 demonstrated that patients who underwent $\mathrm{RC}$ after a major response to chemotherapy (but with residual disease) had survival rates similar to those in patients with a complete response to chemotherapy (with no residual disease) (16). Palliative RC in an attempt to control bleeding and local symptoms is rarely indicated due to the morbidity of the surgery and the limited life expectancy of patients with
mUCB. However, this could change over time as new treatment options, including immune checkpoint blockade, are introduced.

\section{Surgical techniques}

RC entails removal of the bladder, adjacent organs, and regional lymph nodes. In males, it includes the removal of the prostate and the seminal vesicles whereas in females, the reproductive organs (ovaries, fallopian tubes, uterus and anterior vagina) may also be removed to achieve best oncologic outcomes. RC can be distinguished from simple cystectomy in that simple cystectomy is supratrigonal or prostate sparing and often the trigone urothelium along with pelvic lymph nodes is left behind as the bladder is removed. Simple cystectomies are often performed in patients with unrelenting symptoms but not for oncologic control.

\section{Open versus robot assisted $R C$}

Despite advances in technology and patient care, open RC (ORC) still has high rates of perioperative complication and mortality even in the most experienced hands at high volume centers (17). For many abdominal surgeries, the minimally invasive approach is deemed superior with respect to postoperative recovery and peri-operative morbidity without influencing cancer-free rates. With the advent of robotic assisted surgery, there is the potential to improve surgeon precision and dexterity while reducing surgeon fatigue. With this in mind, there have been numerous studies looking to see if robot-assisted RC (RARC) can improve outcomes of this highly morbid surgery. In 2015, Bochner and colleagues reported on a randomized controlled trial comparing ORC and RARC. The trial was closed prematurely at the time of a planned interim analysis when it was apparent that an improvement in the 90-day rate of grade $2-5$ complications with RARC (62\% RARC vs. 66\% ORC) would not be met with further patient enrollment (18). The trial showed decreased blood loss and increased length of surgery, but the length of stay and pathology outcomes were similar and RARC was significantly less cost effective (18).

In the CORAL trial, a single-centre, randomized, controlled three-arm study, Khan and colleagues [2016] aimed to distinguish differences with respect to 30- and 90day complication rates between laparoscopic (LRC), robotic and open cystectomy (19). Secondary end points were 


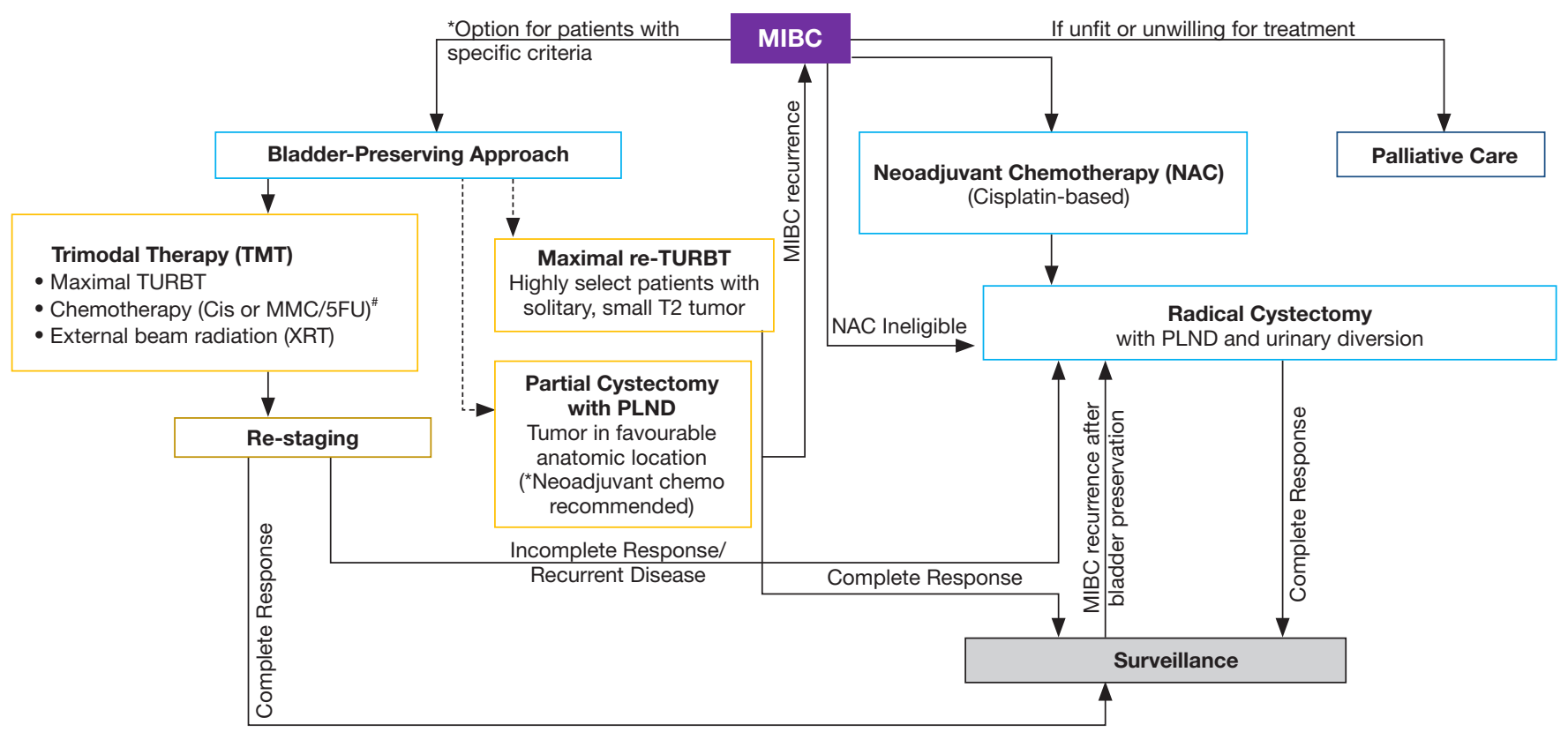

Figure 1 Upon diagnosis of non-metastatic, muscle-invasive bladder cancer, the recommendation is for patients to undergo neo-adjuvant chemotherapy (NAC) followed by radical cystectomy and pelvic lymph node dissection (PLND). Surgery alone is offered to patients ineligible for or declining NAC, radical cystectomy (with possible adjuvant chemotherapy). Trimodal therapy (TMT), as part of a bladderpreserving approach, is another option for select patients. However, if TMT fails, radical cystectomy remains an option for salvage therapy. This figure has been adapted from the AUA guidelines for MIBC therapy (2). *, Option for patients with solitary tumors $\leq 5 \mathrm{~cm}$, absence of hydronephrosis, absence of extensive CIS, good bladder function, or patients who are unfit for or declining cystectomy. * Cis or MMC/5FU = cisplatin or mitomycin $\mathrm{C}$ and 5-fluorouracil.

perioperative clinical, pathologic, and oncologic outcomes, and quality of life (19). Sixty patients were enrolled between 2009 and 2012. The 30-day complication rates were significantly higher in the ORC arm than the LRC arm but there was no significant difference between groups at 90 days (19). Mean operative time was longer in RARC compared with ORC and LRC but there were no significant differences in QoL measures (19). Although the sample size is small in this study, the conclusions do correlate well with the results reported from multiple other non-randomized and retrospective studies.

In the RAZOR trial, Parekh and colleagues randomly assigned 302 patients across 15 US institutions to undergo ORC $(\mathrm{n}=152)$ or RARC $(\mathrm{n}=150)$ between 2011 and 2014 (20). They demonstrated non-inferiority of RARC [72.3\% (95\% CI, 64.3 to 78.8$)]$ in comparison to ORC [71.6\% (95\% CI, 63.6 to 78.2$)]$ with respect to 2 -year progression-free survival (20).

A Cochrane review recently analyzed the results of five randomised controlled trials comprising a total of
541 participants managed by ORC $(\mathrm{n}=270)$ and RARC $(\mathrm{n}=271)(21)$. The primary outcomes included time to recurrence and major complications (Clavien grade 3 to 5). This review concluded that there was little or no difference in oncological outcomes or risk of major complications between RARC and ORC in the treatment of MIBC (21). In this review, they also compared their findings with the findings of other systematic reviews looking at randomized controlled trials and there appeared to be a consensus in the conclusions made by this review and the five other similar studies (21-26). All of these studies were conducted with extracorporeal urinary diversion, which appears to be the key driver of post-operative complications. It remains to be seen if intracorporeal diversion, which is becoming more popular, will change this balance (27). In addition, the robotic platform offers the potential for future technologic enhancements, such as the use of indocyanine green to minimize uretero-enteric strictures, which could lead to more significant improvements in outcomes in the future (28) . 


\section{Pelvic lymph node dissection (PLND)}

PLND has been shown to improve survival and has become a routine element of RC for UCB (29-31). There is, however, still much debate about the optimal extent of PLND for maximal oncologic control. In a meta-analysis by $\mathrm{Ku}$ and colleagues [2015], up to $25 \%$ of organ-confined MIBC had metastatic deposits within the regional lymph nodes (30). Although an extended PLND could potentially help eradicate this metastatic disease, peri-operative complications and cost may increase with an extended template (31).

The nomenclature describing nodal templates varies across studies. A limited template refers to nodes in the perivesical space and obturator fossa, with the external iliac vein as the lateral border and the obturator nerve along the medial border (32). A "standard" lymphadenectomy is proximally bound by the bifurcation of the common iliac artery, extending laterally to the genitofemoral nerve, medially to the bladder and internal iliac vessels and distally by the circumflex iliac vein $(32,33)$. The extended PLND stretches cephalad to the aortic bifurcation (and sometimes above up to the inferior mesentery artery) and includes the pre-sciatic and presacral nodes (34).

Retrospective studies have compared standard and extended PLND. Dhar and colleagues [2008] showed an increase in node-positive disease after an extended PLND (26\%) compared to the limited PNLD group (13\%) across two institutions (32). The 5 -year recurrence-free survival also increased in the extended PLND cohort as compared to the limited PLND (35\% versus 7\%) (32). In a similar comparison involving one of these two cohorts, there was no additional advantage to the "super-extended" template extending up to the inferior mesenteric artery (35). Other retrospective studies have also failed to show any benefit to progression-free survival or overall survival with the superextended PLND $(13,35,36)$.

In several retrospective studies, the number of nodes collected has been associated with improved oncologic outcomes, even in groups who had organ-confined disease with no micrometastatic spread to lymph nodes $(31,37,38)$. There are, however, significant differences in how nodes are counted between institutions and between pathologists, which makes this an imperfect measure $(39,40)$.

A definitive determination of the value of the extended PLND is awaiting the report of a completed phase 3 randomized control trial led by SWOG that randomized $>600$ patients with MIBC to standard versus extended
PLND (41,42). This trial was powered to detect an improvement in 3-year disease-free survival from 55\% to $65 \%$. A similar but smaller German trial recently demonstrated no advantage to the extended PLND (43). This study enrolled 401 patients and included also patients with T1 disease. A combined analysis of both trials may be necessary to detect a more subtle difference in oncologic outcome.

\section{Urinary diversion}

Urinary diversion is a necessary component of RC. Two general categories of diversion can be distinguished: incontinent and continent diversion (44).

Incontinent diversions are more widely used as they are technically easier to perform and are more easily managed by the elderly, frail RC population $(44,45)$. The simplest diversion is the cutaneous ureterostomy, which, however, fell out of favor due to the high risk of stricture of the ostomy sites (46). The advantage of this strategy is the avoidance of a bowel anastomosis, which reduces the risk of complications, especially in patients who may otherwise be marginal for RC. The cutaneous ureterostomy is therefore sometimes still performed in frail patients at select centers (47). Similarly, the ureterosigmoidostomy was a simple method for urinary diversion, but it too has fallen out of favor due to the risk of pyelonephritis and adenocarcinoma at the site of ureteral implantation into the colon $(37,38)$.

Ileal conduits were the benchmark for urinary diversion before continent diversions were described and remain the procedure of choice especially in older patients and patients with significant other comorbidities (46). Reported complication rates with ileal conduits range from $45 \%$ at 5 years to $94 \%$ at 15 years and they include renal insufficiency, stomal complications including parastomal hernias and stomal stenosis, urinary tract infections, ureteral obstruction and stone formation $(46,48)$.

The continent diversion is further divided into continent cutaneous diversion and orthotopic neobladder. Absolute contraindications to continent diversion include poor renal function, severe hepatic dysfunction, and significant inflammatory bowel disease (39). Continent diversions have a higher rate of metabolic complications through reabsorption of waste products (49). Relative contraindications include previous pelvic and abdominal radiation and mental impairment (50). Malignant 
involvement of the urethra requiring urethrectomy and recurrent urethral strictures represent contraindications for neobladder but not continent cutaneous diversion (50).

Patients with a continent cutaneous diversion $(46,51)$ are committed to lifelong intermittent self-catheterization. With reported daytime and nighttime continence rates of $90 \%$ to $98 \%$, this method of reconstruction is associated with a good quality of life post RC in most patients $(52,53)$. Potential complications include stomal stenosis requiring reoperation in as many as $21 \%$ of patients (54), and stone formation $(46,48,54)$. This technique is less frequently used with the advent of the orthotopic urinary diversion.

Voiding of the orthotopic neobladder is accomplished via relaxation of the external sphincter combined with the Valsalva maneuver $(50,55)$. Daytime continence rates exceed $85 \%$ at high volume centers but do vary between $47 \%$ to $100 \%$ depending on the definition of continence and the length of follow-up (56-58). Up to $50 \%$ of patients have nocturnal incontinence due to loss of physiological storage reflexes. Higher rates of ventral hernias may be observed after neobladder construction due to the use of Valsalva voiding (50). Intermittent self-catheterization after neobladder surgery is required due to incomplete bladder emptying in approximately $4 \%$ to $25 \%$ of patients based on a meta-analysis of more than 2,000 patients and is significantly more predominant in women (59).

Choosing the type of diversion and reconstruction is a decision that has to be made based on a variety of factors including cancer control and patient comorbidities, as well as surgical suitability, post-operative ability to manage the diversion care, and patient preference (60). Few formal studies have documented a difference in the overall quality of life between incontinent or continent urinary diversion, although all such studies are limited by the simple fact that the patient usually will have selected his or her preference for diversion (61-63). A systematic review of health-related quality of life failed to show a superiority of any of the techniques of urinary diversion in quality of life (64).

\section{Complications of RC}

$\mathrm{RC}$ is associated with relatively high perioperative morbidity and mortality, despite improvements in surgical techniques and peri-operative care (17). The incidence of complications after surgery has been reported to be in the range of $30-70 \%(49,57,65,66)$. There is a large number of single center studies that document either surgeon specific or center specific results and a number of national databases which have combined such reported results and those show a consistent trend among centers with regard to morbidity and mortality despite the absence of a formal complication reporting system $(17,67)$.

Complications related to $\mathrm{RC}$ can be divided into early and late, with early complications referring to those that occur within 30 days of the operation and late encompassing complications occurring between 30-90 days $(17,23,68)$. Postoperative mortality rates range from $0.8 \%$ to up to $3 \%(23,68,69)$. Mortality appears to be lower in high-volume academic centers even though the patient population at these centers is enriched for those with more co-morbidities (65).

Knap and colleagues [2004] reported common late complications to include ventral hernia (17\%), calculus formation (16\%), ureteroenteric strictures (14\%) and parastomal hernias (8\%). The authors also determined that vitamin B12 deficiency and loss of kidney function were more common in older than younger patients. Fifty percent of patients over 70 years experienced (70). Similarly, $26 \%$ of patients over the age of 70 experienced loss of renal function in five-year follow-up as compared to $7 \%$ of patients under the age of 70 (70).

Shabsigh and colleagues [2009] provided one of the first systematic reports of post RC complications using the standardized Clavien-Dindo classification. They analysed data from 1,142 consecutive cystectomies between 1995 and 2005 and found that 735 (64\%) experienced a complication within 90 days of surgery (17). The majority of these (79\%) were grade 1 to 2 and considered to be "minor". Most patients $(58 \%)$ had one or more complication within the first 30 days after surgery (17).

The most common complication categories were gastrointestinal (29\%), infectious (25\%), wound-related $(15 \%)$, cardiac $(11 \%)$, and genitourinary $(11 \%)$; however, this pattern did change from early to the late phase of complications. The most common early all-grade complications were gastrointestinal, infectious and woundrelated. This switched to infectious, gastrointestinal and genitourinary in the late phase. It must be noted, however, that in both early and late phase, the most common highgrade complications were genitourinary (17). Similar findings were made in another large series (71).

An important advance in our efforts to reduce perioperative complications and improve post-operative quality of life has been the implementation of Enhanced Recovery after Surgery (ERAS) pathways that define numerous evidence-based standard interventions to be followed from 
pre-operative education to intra-operative fluid management and post-operative nutrition $(72,73)$. Although the impact of any one element is often uncertain, there is compelling evidence that the cumulation of elements shortens hospitals stays and reduces the rate of complications $(74,75)$.

Going forward, it is important to develop a system of unified reporting with cystectomy-related complications because this data can be used to assess pre-operative risk of individual patients for developing post-cystectomy complications $(17,68,76,77)$. If patients can be optimized pre-operatively or be offered other methods of management if the risk of an operation is prohibitive $(16,49,60)$. Careful patient selection is key to a successful operation.

\section{Conclusions}

$\mathrm{RC}$ plays a central role in the multimodal therapy of patients with high risk NMIBC and MIBC. The negative impact on quality of life and the high rate of peri-operative complications pose important limitations, especially in the context of a disease that affects primarily elderly patients with multiple medical co-morbidities. RC must therefore be viewed as one element of a complex, multidisciplinary treatment plan. Measures to improve outcomes include regionalization of care to high volume centers, the use of ERAS protocols, and technologic advances such as robotic surgery, although this last element has yet to have a major impact on outcomes. Patient selection and the tailoring of the urinary diversion to the patient's needs are paramount. Level I evidence to direct the use of the extended PLND is eagerly awaited in the near future, and neoadjuvant therapies are likely to evolve with advances in systemic therapy. These are all meaningful strides in the effort to improve patient outcomes.

\section{Acknowledgments}

Funding: None.

\section{Footnote}

Provenance and Peer Review: This article was commissioned by the Guest Editor (Shomik Sengupta) for the series "Surgery for Urologic Cancers" published in Translational Andrology and Urology. The article has undergone external peer review.

Conflicts of Interest: Both authors have completed the
ICMJE uniform disclosure form (available at http://dx.doi. org/10.21037/tau.2020.03.23). The series "Surgery for Urologic Cancers" was commissioned by the editorial office without any funding or sponsorship. Dr. Black reports grants from iProgen, Sanofi, Bayer, GSK, outside the submitted work; In addition, Dr. Black has a patent Decipher Bioscience pending and A member of a Speakers bureau: AbbVie, Biosyent, Janssen, Ferring, TerSera, Pfizer; he is currently participating in or has participated in a clinical trial within the past two years, including Genentech, Janssen, BMS, Astellas, Sitka, MDx Health, AstraZeneca, Therelase, Pacific Edge. Dr. Aminoltejari has no other conflicts of interest to declare.

Ethical Statement: The authors are accountable for all aspects of the work in ensuring that questions related to the accuracy or integrity of any part of the work are appropriately investigated and resolved.

Open Access Statement: This is an Open Access article distributed in accordance with the Creative Commons Attribution-NonCommercial-NoDerivs 4.0 International License (CC BY-NC-ND 4.0), which permits the noncommercial replication and distribution of the article with the strict proviso that no changes or edits are made and the original work is properly cited (including links to both the formal publication through the relevant DOI and the license). See: https://creativecommons.org/licenses/by-nc-nd/4.0/.

\section{References}

1. American Cancer Society. Cancer Facts \& Figures 2019. 2019.

2. Chang SS, Bochner BH, Chou R, et al. Treatment of NonMetastatic Muscle-Invasive Bladder Cancer: AUA/ASCO/ ASTRO/SUO Guideline. J Urol 2017;198:552-9.

3. Gore JL, Litwin MS, Lai J, et al. Use of radical cystectomy for patients with invasive bladder cancer. J Natl Cancer Inst 2010;102:802-11.

4. Stein JP, Lieskovsky G, Cote R, et al. Radical cystectomy in the treatment of invasive bladder cancer: long-term results in 1,054 patients. J Clin Oncol 2001;19:666-75.

5. Williams SB, Hudgins HK, Ray-Zack MD, et al. Systematic Review of Factors Associated with the Utilization of Radical Cystectomy for Bladder Cancer. Eur Urol Oncol 2019;2:119-25.

6. Hautmann RE, Gschwend JE, de Petriconi RC, et al. Cystectomy for transitional cell carcinoma of the bladder: 
results of a surgery only series in the neobladder era. J Urol 2006;176:486-92; discussion 491.

7. Stein JP, Skinner DG. Radical cystectomy for invasive bladder cancer: long-term results of a standard procedure. World J Urol 2006;24:296-304.

8. Tyritzis SI, Wiklund NP. Is the open cystectomy era over? An update on the available evidence. Int J Urol 2018;25:187-95.

9. Siefker-Radtke AO, Apolo AB, Bivalacqua TJ, et al. Immunotherapy with Checkpoint Blockade in the Treatment of Urothelial Carcinoma. J Urol 2018;199:1129-42.

10. US Department of Health and Human Services Food and Drug Administration. BCG-Unresponsive Non-muscle Invasive Bladder Cancer: Developing Drugs and Biologics for Treatment Guidance for Industry. 2019; Accessed 10/31, 2019.

11. Kirkali Z, Chan T, Manoharan M, et al. Bladder cancer: epidemiology, staging and grading, and diagnosis. Urology 2005;66:4-34.

12. Power NE, Izawa J. Comparison of Guidelines on NonMuscle Invasive Bladder Cancer (EAU, CUA, AUA, NCCN, NICE). Bladder Cancer 2016;2:27-36.

13. Mak RH, Hunt D, Shipley WU, et al. Long-term outcomes in patients with muscle-invasive bladder cancer after selective bladder-preserving combined-modality therapy: a pooled analysis of Radiation Therapy Oncology Group protocols 8802, 8903, 9506, 9706, 9906, and 0233. J Clin Oncol 2014;32:3801-9.

14. James ND, Hussain SA, Hall E, et al. Radiotherapy with or without chemotherapy in muscle-invasive bladder cancer. N Engl J Med 2012 ;366:1477-88.

15. Gray PJ, Fedewa SA, Shipley WU, et al. Use of potentially curative therapies for muscle-invasive bladder cancer in the United States: results from the National Cancer Data Base. Eur Urol 2013;63:823-9.

16. Dodd PM, McCaffrey JA, Herr H, et al. Outcome of postchemotherapy surgery after treatment with methotrexate, vinblastine, doxorubicin, and cisplatin in patients with unresectable or metastatic transitional cell carcinoma. J Clin Oncol 1999;17:2546-52.

17. Shabsigh A, Korets R, Vora KC, et al. Defining early morbidity of radical cystectomy for patients with bladder cancer using a standardized reporting methodology. Eur Urol 2009;55:164-74.

18. Bochner BH, Dalbagni G, Sjoberg DD, et al. Comparing Open Radical Cystectomy and Robot-assisted Laparoscopic Radical Cystectomy: A Randomized Clinical
Trial. Eur Urol 2015;67:1042-50.

19. Khan MS, Gan C, Ahmed K, et al. A Single-centre Early Phase Randomised Controlled Three-arm Trial of Open, Robotic, and Laparoscopic Radical Cystectomy (CORAL). Eur Urol 2016;69:613-21.

20. Parekh DJ, Reis IM, Castle EP, et al. Robot-assisted radical cystectomy versus open radical cystectomy in patients with bladder cancer (RAZOR): an open-label, randomised, phase 3, non-inferiority trial. Lancet 2018;391:2525-36.

21. Rai BP, Bondad J, Vasdev N, et al. Robotic versus open radical cystectomy for bladder cancer in adults. Cochrane Database Syst Rev 2019;4:CD011903.

22. Yuh B, Wilson T, Bochner B, et al. Systematic review and cumulative analysis of oncologic and functional outcomes after robot-assisted radical cystectomy. Eur Urol 2015;67:402-22.

23. Novara G, Catto JW, Wilson T, et al. Systematic review and cumulative analysis of perioperative outcomes and complications after robot-assisted radical cystectomy. Eur Urol 2015;67:376-401.

24. Ishii H, Rai BP, Stolzenburg JU, et al. Robotic or open radical cystectomy, which is safer? A systematic review and meta-analysis of comparative studies. J Endourol 2014;28:1215-23.

25. Sathianathen NJ, Kalapara A, Frydenberg M, et al. Robotic Assisted Radical Cystectomy vs Open Radical Cystectomy: Systematic Review and Meta-Analysis. J Urol 2019;201:715-20.

26. Tang K, Xia D, Li H, et al. Robotic vs. open radical cystectomy in bladder cancer: A systematic review and meta-analysis. Eur J Surg Oncol 2014;40:1399-411.

27. Zhang JJH, Ericson KJ, Thomas LJ, et al. Large SingleInstitution Comparison of Perioperative Outcomes and Complications in Open Radical Cystectomy, Intracorporeal Robot-Assisted Radical Cystectomy, and Robotic Extracorporeal Approach. J Urol 2019:101097JU0000000000000570.

28. Ahmadi N, Ashrafi AN, Hartman N, et al. Use of indocyanine green to minimise uretero-enteric strictures after robotic radical cystectomy. BJU Int 2019;124:302-7.

29. Vieweg J, Gschwend JE, Herr HW, et al. The impact of primary stage on survival in patients with lymph node positive bladder cancer. J Urol 1999;161:72-6.

30. Ku JH, Kang M, Kim HS, et al. Lymph node density as a prognostic variable in node-positive bladder cancer: a meta-analysis. BMC Cancer 2015;15:447.

31. Perera M, McGrath S, Sengupta S, et al. Pelvic lymph node dissection during radical cystectomy for muscle- 
invasive bladder cancer. Nat Rev Urol 2018;15:686-92.

32. Dhar NB, Klein EA, Reuther AM, et al. Outcome after radical cystectomy with limited or extended pelvic lymph node dissection. J Urol 2008;179:873-8; discussion 878.

33. Konety BR, Joslyn SA, O'Donnell MA. Extent of pelvic lymphadenectomy and its impact on outcome in patients diagnosed with bladder cancer: analysis of data from the Surveillance, Epidemiology and End Results Program data base. J Urol 2003;169:946-50.

34. Poulsen AL, Horn T, Steven K. Radical cystectomy: extending the limits of pelvic lymph node dissection improves survival for patients with bladder cancer confined to the bladder wall. J Urol 1998;160:2015-9; discussion 2020.

35. Zehnder P, Studer UE, Skinner EC, et al. Super extended versus extended pelvic lymph node dissection in patients undergoing radical cystectomy for bladder cancer: a comparative study. J Urol 2011;186:1261-8.

36. Stein JP. Lymphadenectomy in bladder cancer: how high is "high enough"? Urol Oncol 2006;24:349-55.

37. Crozier J, Papa N, Perera M, et al. Lymph node yield in node-negative patients predicts cancer specific survival following radical cystectomy for transitional cell carcinoma. Investig Clin Urol 2017;58:416-22.

38. May M, Herrmann E, Bolenz C, et al. Association between the number of dissected lymph nodes during pelvic lymphadenectomy and cancer-specific survival in patients with lymph node-negative urothelial carcinoma of the bladder undergoing radical cystectomy. Ann Surg Oncol 2011;18:2018-25.

39. Dorin RP, Daneshmand S, Eisenberg MS, et al. Lymph node dissection technique is more important than lymph node count in identifying nodal metastases in radical cystectomy patients: a comparative mapping study. Eur Urol 2011;60:946-52.

40. Metcalfe MJ, Afshar K, So AI, et al. A standardized protocol for identifying and counting lymph nodes harvested by pelvic lymph node dissection at the time of radical cystectomy. Can Urol Assoc J 2015;9:337-42.

41. ClinicalTrials gov. US National Library of Medicine. 2019; Available online: https://clinicaltrials.gov/ct2/show/ NCT01224665. Accessed 09/12, 2019.

42. US National Library of Medicine. ClinicalTrials.gov. 2018; Available online: https://clinicaltrials.gov/ct2/show/ NCT01215071. Accessed 09/12, 2019.

43. Gschwend JE, Heck MM, Lehmann J, et al. Extended Versus Limited Lymph Node Dissection in Bladder Cancer Patients Undergoing Radical Cystectomy: Survival
Results from a Prospective, Randomized Trial. Eur Urol 2019;75:604-11.

44. Razik A, Das CJ, Gupta A, et al. Urinary diversions: a primer of the surgical techniques and imaging findings. Abdom Radiol (NY) 2019;44:3906-18.

45. Madersbacher S, Schmidt J, Eberle JM, et al. Longterm outcome of ileal conduit diversion. J Urol 2003;169:985-90.

46. Farnham SB, Cookson MS. Surgical complications of urinary diversion. World J Urol 2004;22:157-67.

47. Longo N, Imbimbo C, Fusco F, et al. Complications and quality of life in elderly patients with several comorbidities undergoing cutaneous ureterostomy with single stoma or ileal conduit after radical cystectomy. BJU Int 2016;118:521-6.

48. Kouba E, Sands M, Lentz A, et al. Incidence and risk factors of stomal complications in patients undergoing cystectomy with ileal conduit urinary diversion for bladder cancer. J Urol 2007;178:950-4.

49. Konety BR, Allareddy V, Herr H. Complications after radical cystectomy: analysis of population-based data. Urology 2006;68:58-64.

50. Moon A, Vasdev N, Thorpe AC. Continent urinary diversion. Indian J Urol 2013;29:303-9.

51. Levy ME, Elliott SP. Reconstructive techniques for creation of catheterizable channels: tunneled and nipple valve channels. Transl Androl Urol 2016;5:136-44.

52. Kaefer M, Retik AB. The Mitrofanoff principle in continent urinary reconstruction. Urol Clin North Am 1997;24:795-811.

53. López Pereira P, Velasco B, Martinez Urrutia MJ, et al. The Mitrofanoff principle in the lower urinary tract reconstruction. Cir Pediatr 1997;10:29-33.

54. Holmes DG, Thrasher JB, Park GY, et al. Long-term complications related to the modified Indiana pouch. Urology 2002;60:603-6.

55. Lee KS, Montie JE, Dunn RL, et al. Hautmann and Studer orthotopic neobladders: a contemporary experience. J Urol 2003;169:2188-91.

56. Studer UE, Danuser H, Hochreiter W, et al. Summary of 10 years' experience with an ileal low-pressure bladder substitute combined with an afferent tubular isoperistaltic segment. World J Urol 1996;14:29-39.

57. Hautmann RE, de Petriconi R, Gottfried HW, et al. The ileal neobladder: complications and functional results in 363 patients after 11 years of followup. J Urol 1999;161:422-7; discussion 427.

58. Steven K, Poulsen AL. The orthotopic Kock ileal 
neobladder: functional results, urodynamic features, complications and survival in 166 men. J Urol 2000;164:288-95.

59. Steers WD. Voiding dysfunction in the orthotopic neobladder. World J Urol 2000;18:330-7.

60. McAlpine K, Lavallee LT, Stacey D, et al. Development and Acceptability Testing of a Patient Decision Aid for Urinary Diversion with Radical Cystectomy. J Urol 2019;202:1001-7.

61. Dutta SC, Chang SC, Coffey CS, et al. Health related quality of life assessment after radical cystectomy: comparison of ileal conduit with continent orthotopic neobladder. J Urol 2002;168:164-7.

62. Hobisch A, Tosun K, Kinzl J, et al. Quality of life after cystectomy and orthotopic neobladder versus ileal conduit urinary diversion. World J Urol 2000;18:338-44.

63. Henningsohn L, Steven K, Kallestrup EB, et al. Distressful symptoms and well-being after radical cystectomy and orthotopic bladder substitution compared with a matched control population. J Urol 2002;168:16874; discussion 174.

64. Porter MP, Penson DF. Health related quality of life after radical cystectomy and urinary diversion for bladder cancer: a systematic review and critical analysis of the literature. J Urol 2005;173:1318-22.

65. Jerlström T, Gardmark T, Carringer M, et al. Urinary bladder cancer treated with radical cystectomy: perioperative parameters and early complications prospectively registered in a national population-based database. Scand J Urol 2014;48:334-40.

66. Hautmann RE, de Petriconi RC, Volkmer BG. Lessons learned from 1,000 neobladders: the 90-day complication rate. J Urol 2010;184:990-4; quiz 1235.

67. Chang SS, Cookson MS, Baumgartner RG, et al. Analysis of early complications after radical cystectomy: results of a collaborative care pathway. J Urol 2002;167:2012-6.

68. Lauridsen SV, Tonnesen H, Jensen BT, et al.

Cite this article as: Aminoltejari K, Black PC. Radical cystectomy: a review of techniques, developments and controversies. Transl Androl Urol 2020;9(6):3073-3081. doi: $10.21037 /$ tau.2020.03.23
Complications and health-related quality of life after robot-assisted versus open radical cystectomy: a systematic review and meta-analysis of four RCTs. Syst Rev 2017;6:150.

69. Takada N, Abe T, Shinohara N, et al. Peri-operative morbidity and mortality related to radical cystectomy: a multi-institutional retrospective study in Japan. BJU Int 2012;110:E756-64.

70. Knap MM, Lundbeck F, Overgaard J. Early and late treatment-related morbidity following radical cystectomy. Scand J Urol Nephrol 2004;38:153-60.

71. Smith JA. Early and late treatment-related morbidity following radical cystectomy. J Urol 2005;173:2033-4.

72. Collins JW, Patel H, Adding C, et al. Enhanced Recovery After Robot-assisted Radical Cystectomy: EAU Robotic Urology Section Scientific Working Group Consensus View. Eur Urol 2016;70:649-60.

73. Cerantola Y, Valerio M, Persson B, et al. Guidelines for perioperative care after radical cystectomy for bladder cancer: Enhanced Recovery After Surgery (ERAS(®)) society recommendations. Clin Nutr 2013;32:879-87.

74. Daneshmand S, Ahmadi H, Schuckman AK, et al. Enhanced recovery protocol after radical cystectomy for bladder cancer. J Urol 2014;192:50-5.

75. Frees SK, Aning J, Black P, et al. A prospective randomized pilot study evaluating an ERAS protocol versus a standard protocol for patients treated with radical cystectomy and urinary diversion for bladder cancer. World J Urol 2018;36:215-20.

76. Mannas MP, Lee T, Forbes CM, et al. Predicting complications following radical cystectomy with the ACS NSQIP universal surgical risk calculator. World J Urol 2020;38:1215-20.

77. Janisch F, Yu H, Vetterlein MW, et al. Do Younger Patients with Muscle-Invasive Bladder Cancer have Better Outcomes? J Clin Med 2019;8:1459. 